\title{
Study Regarding Conceptualisation of Dermatology Operation Theatre Based on Perception of the Five Human Senses
}

Sundeep Chowdhry MBBS ${ }^{1}$, MD, Prashansa Jaiswal, MBBS², MD, DNB*, Paschal D' Souza MBBS, MD ${ }^{3}$

${ }^{1}$ Senior Specialist and Assistant Professor, Department of Dermatology, Venereology and Leprology ESIPGIMSR, Basaidarapur, New Delhi. ${ }^{2}$ Diplomat in Dermatology (Glasgow), Department of Dermatology, Venereology and Leprology, ESIPGIMSR, Basaidarapur, New Delhi, India. ${ }^{3}$ Director Professor, Department of Dermatology, Venereology and Leprology ESIPGIMSR, Basaidarapur, New Delhi.

*Corresponding Author: Dr.Prashansa Jaiswal, MBBS, MD, DNB, Diplomat in Dermatology (Glasgow), Department of Dermatology, Venereology and Leprology, ESIPGIMSR, Basaidarapur, New Delhi, India. Address: A2 311, Sunrise Apartment, Sector 13, Rohini, New Delhi110085 .

Received date: July 09, 2020; Accepted date: October 17, 2020; Published date: October 23, 2020

Citation: S Chowdhry, P Jaiswal, P D Souza. (2020) Study Regarding Conceptualisation of Dermatology Operation Theatre Based on Perception of the Five Human Senses. Journal of Dermatology and Dermatitis.5 (2); Doi: 10.31579/2578-8949/070

Copyright: (92020 Prashansa Jaiswal, This is an open-access article distributed under the terms of The Creative Commons. Attribution License, which permits unrestricted use, distribution, and reproduction in any medium, provided the original author and source are credited.

\begin{abstract}
Background: In general a pleasant enviornment has a positive effect on the occupant's feeling of well-being and this can affect patient recovery and staff performance. Therefore lighting, colour design, aroma and good behaviour of doctors are very essential Based on these ideas we conducted a study in our hospital premises as to how our 5 senses can help to conceptualise a dermatology operation theatre.
\end{abstract}

Method: The study was carried out in the Department of Dermatology, Employees State Insurance - Post Graduate Institute of Medical Sciences \& Research (ESI-PGIMSR), Basaidarapur, New Delhi. 120 was the sample size which was divided in to 3 groups of doctors, nurses and patients of 40 each. All cases were evaluated using case record proforma comprising of their demographic profile and 21 questions which was prevalidated.

Results: In choosing colour of wall paint, $60 \%$ doctors opted white and $32.5 \%$ yellow. $53.3 \%$ selected white in total followed by yellow $21.7 \%$ followed by blue $15.8 \%$ and vibrant colour $9.2 \%$. While choosing colour of linen (bed sheets) most doctors chose green colour (55\%). All (100\%) preferred provision of windows/ ventilator in OT for natural light. Hearing calming words by doctors before surgery was appreciated by all nurses and patients $(100 \%)$.

Conclusion: Five senses of human body play an important role while conceptualising the operation theatre.

Keywords- five senses; colour psychology; dermatology operation theatre; doctor's behaviour

\section{Introduction}

The quality of the visual environment has a positive effect on the occupant's feeling of well-being and in the case of hospitals and healthcare buildings this can affect patient recovery and staff performance. For these reasons, it is essential to consider lighting and colour design at the early stages of specifying and designing a building. The colour and materials of window treatments such as blinds, curtains or upholstery needs to be chosen with care so as to enhance the interiors or control glare. Hence, a properly designed visually soothing environment, with the appropriate use of colour and lighting, will give necessary benefits to patients [1]. Similarly, behaviour of doctors towards their patients is also very important and is at par with their clinical skills. His/her good and polite behaviour is an important component of quality health care. It helps in allaying nervousness and anxiety of the patient [2]. Aromas have different effects on everyone. Smells can affect both our short and long term moods. Creating an environment with pleasant smells evoke positive, calm and good emotions [3]. An emotional response of the secretion of immune-boosting hormones is induced by certain music
[4]. Based on these ideas we conducted a study in our hospital premises as to how our 5 senses can help to conceptualise a dermatology operation theatre.

\section{Aim of the Study}

The aim of the study was to conceptualise dermatology operation theatre based on perception of the five human senses using set of questionnaire.

\section{Method and Material}

The study was carried out in the Department of Dermatology, Employees State Insurance - Post Graduate Institute of Medical Sciences \& Research (ESI-PGIMSR), Basaidarapur, New Delhi after the ethical clearance from the institutional ethics committee. It was a cross sectional study. 120 was the sample size which was divided in to 3 groups of doctors, nurses and patients of 40 each. All cases were evaluated using case record proforma (Table 1) comprising of their demographic profile and 21 questions in the questionnaire which was prevalidated. The questionnaire was answered comprehensively. 
Age:

Sex: $\mathrm{M} / \mathrm{F}$

Patients/Nurse/doctor

1. Which colour of wall paint would you prefer to be used in dermatology OT? White/ blue/ green/ black/ vibrant colour.

2. Which colour of drapes would you prefer to be used in dermatology OT? White/ blue/ green/ black/ vibrant colour.

3. Which colour of curtain would you prefer to visualise in surrounding in dermatology OT? White/ blue/ green/ black/ vibrant colour.

4. Which colour of linen (bed sheets) would you prefer on the operating table in dermatology OT? White/ blue/ green/ black/ vibrant colour.

5. Which colour of furniture including cabinets / laminates would you prefer to be used in dermatology OT? White/blue/green/black/vibrant colour.

6. Would you prefer to have provision of windows or ventilator in dermatology OT for natural light? Yes/No

7. Does display of instruments in transparent cupboard shelves or kept openly on slabs /shelves in dermatology OT generate apprehension on visualising the same? Yes/No

8. Is your nervousness and anxiety allayed on visualising a greeting smile on the face of treating dermatosurgeon? Yes / No

9. Which fragrance / room freshener is preferred by you to be used in the OT room? Floral / Cologne / Fruity / Any Other

10. Do you prefer to avoid the characteristic hospital smell/smell of latex glove/smell of antiseptics and medications, if given a choice? Yes / No

11. Would you like to hear soothing, pleasantries; calming words prior to procedure by doctor/supporting OT staff? Yes/No

12. Which type / nature of background music will impart a soothing effect on your mind prior and during the operative procedure? Silence)/classical/soft/instrumental/soft vocal/jazz beats/any specific music.

13. Does behaviour, general non-specific mutual discussion, unnecessary casual comments, lack of apparent concern or negative work attitude of supporting staff cause apprehension in your mind during the procedure? Yes/No

14. Before entering the OT which light refreshment(s) would you prefer prior to procedure? Water/tea/coffee/juice/soft drink/any other

15. Do you prefer to eat any refreshment or snack in post-operative period - Yes / No

16. Do you prefer a dehumified, cool and pleasant environment rather than hot and humid environment, which is usually found in tropical country like India? Yes/No

17. Do you prefer additional presence of fan in air conditioned room for fresh blow of air to avoid feeling of suffocation? Yes/No

18. Do you prefer to avoid excessive use of topical chemicals and medications during the procedure, apart from those absolutely necessary for the said procedure on your skin - Yes / No

19. Do you feel discomfort when instruments, surgical instruments are kept either on the abdomen or other body parts over the drapes during the surgical procedure - Yes / No

20. During procedure which position do you find more comfortable? Sitting on chair/ Lying on table

21. While undergoing procedure which position do you find more comfortable? Supine/prone/lateral

Table 1: Questionnaire for the study

\section{Results}

1. Which colour of wall paint would you prefer to be used in dermatology OT? White/ blue/ green/ black/ vibrant colour. Crosstab

\begin{tabular}{|c|c|c|c|c|c|}
\hline & & \multicolumn{3}{|c|}{ TYPE OF OBSERVER } & \multirow[t]{2}{*}{ Total } \\
\hline & & 1 & 2 & 3 & \\
\hline BLUE & $\begin{array}{l}\text { Count } \\
\% \text { within TYPE OF OBSERVER }\end{array}$ & $\begin{array}{l}3 \\
7.5 \%\end{array}$ & $\begin{array}{l}9 \\
22.5 \%\end{array}$ & $\begin{array}{l}7 \\
17.5 \%\end{array}$ & $\begin{array}{l}19 \\
15.8 \%\end{array}$ \\
\hline \multirow{2}{*}{ PINK } & Count & 0 & 11 & 0 & 11 \\
\hline & $\%$ within TYPE OF OBSERVER & $0.0 \%$ & $27.5 \%$ & $0.0 \%$ & $9.2 \%$ \\
\hline \multirow{2}{*}{ WHITE } & Count & 24 & 17 & 23 & 64 \\
\hline & $\%$ within TYPE OF OBSERVER & $60.0 \%$ & $42.5 \%$ & $57.5 \%$ & $53.3 \%$ \\
\hline \multirow{4}{*}{ YELLOW } & Count & 13 & & 10 & 26 \\
\hline & $\%$ within TYPE OF OBSERVER & $32.5 \%$ & $7.5 \%$ & $25.0 \%$ & $21.7 \%$ \\
\hline & Count & & & 40 & 120 \\
\hline & $\%$ within TYPE OF OBSERVER & $100.0 \%$ & $100.0 \%$ & $100.0 \%$ & $100.0 \%$ \\
\hline
\end{tabular}

Chi-Square Tests

\begin{tabular}{|l|l|l|l|}
\hline & Value & df & $\begin{array}{l}\text { Asymp. Sig. (2- } \\
\text { sided) }\end{array}$ \\
\hline $\begin{array}{l}\text { Pearson Chi-Square } \\
\text { Likelihood Ratio } \\
\text { N of Valid Cases }\end{array}$ & $\begin{array}{l}32.368^{\mathrm{a}} \\
35.845\end{array}$ & 6 & .000 \\
120 & 6 & .000 \\
\hline
\end{tabular}

a. 3 cells $(25.0 \%)$ have expected count less than 5 . The minimum expected count is 3.67 . 
In choosing colour of wall paint in dermatology operation theatre, $7.5 \%$ doctors opted blue, $60 \%$ white and $32.5 \%$ opted for yellow. Among nurses $22.5 \%$ opted blue, white was chosen by $42.5 \%$ and yellow by $7.5 \%$. Among patients $53.3 \%$ chose white followed by yellow $21.7 \%$ followed by blue $15.8 \%$ followed by vibrant colour that was pink $9.2 \%$. Thus, $53.3 \%$ selected white in total followed by yellow $21.7 \%$ followed by blue $15.8 \%$ and vibrant colour $9.2 \%$. Result was significant (.000)

\section{Which colour of drapes would you prefer to be used in dermatology OT? White/ blue/ green/ black/ vibrant colour.}

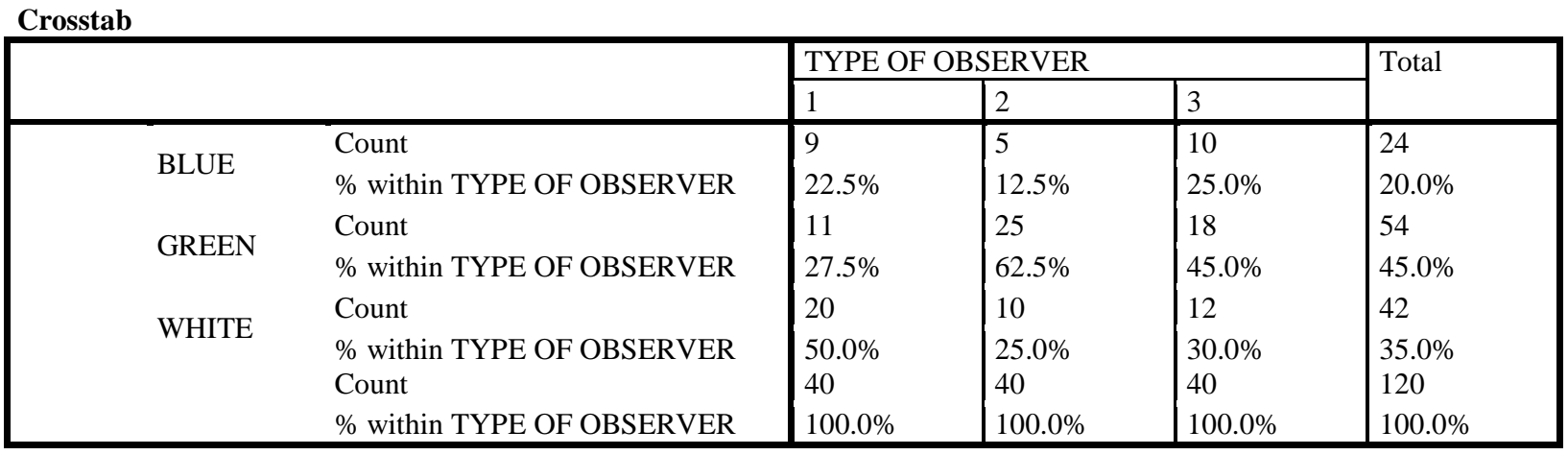

Chi-Square Tests

\begin{tabular}{|l|l|l|l|}
\hline & Value & df & $\begin{array}{l}\text { Asymp. Sig. (2- } \\
\text { sided) }\end{array}$ \\
\hline $\begin{array}{l}\text { Pearson Chi-Square } \\
\text { Likelihood Ratio }\end{array}$ & $11.194^{\mathrm{a}}$ & 4 & .024 \\
N of Valid Cases & 11.312 & 4 & .023 \\
\hline
\end{tabular}

a. 0 cells $(0.0 \%)$ have expected count less than 5 . The minimum expected count is 8.00 .

For colour of drapes $22.5 \%$ doctors' opted blue, $27.5 \%$ green. While most $50 \%$ opted for whilte. Among nurses $12.5 \%$ opted blue, green was chosen by $62.5 \%$ and white by $25 \%$. In patients $25 \%$ chose blue $45 \%$ green and $30 \%$ white. While choosing colour of drapes green was most common $45.0 \%$ followed by white $35 \%$ and blue $20 \%$. Among all colours black and vibrant colour were not selected. Result was not significant.

3. Which colour of curtain would you prefer to visualise in surrounding in dermatology OT? White/ blue/ green/ black/ vibrant colour. Crosstab

\begin{tabular}{|cl|l|l|l|l|}
\hline & & \multicolumn{2}{l|}{ TYPE OF OBSERVER } & Total \\
\cline { 3 - 5 } & & 1 & 2 & 3 & \\
\hline \multirow{2}{*}{ BLUE } & Count & 10 & 25 & 21 & 56 \\
& \% within TYPE OF OBSERVER & $25.0 \%$ & $62.5 \%$ & $52.5 \%$ & $46.7 \%$ \\
& Count & 5 & 3 & 1 & 9 \\
\multirow{2}{*}{ VIBRANT } & $\%$ within TYPE OF OBSERVER & $12.5 \%$ & $7.5 \%$ & $2.5 \%$ & $7.5 \%$ \\
& Count & 25 & 12 & 18 & 55 \\
& \% within TYPE OF OBSERVER & $62.5 \%$ & $30.0 \%$ & $45.0 \%$ & $45.8 \%$ \\
& Count & 40 & 40 & 40 & 120 \\
& \% within TYPE OF OBSERVER & $100.0 \%$ & $100.0 \%$ & $100.0 \%$ & $100.0 \%$ \\
\hline
\end{tabular}

Chi-Square Tests

\begin{tabular}{|c|c|c|c|}
\hline & Value & df & \begin{tabular}{|lll}
$\begin{array}{l}\text { Asymp. } \\
\text { sided })\end{array}$ & Sig. \\
\end{tabular} \\
\hline $\begin{array}{l}\text { Pearson Chi-Square } \\
\text { Likelihood Ratio } \\
\text { N of Valid Cases }\end{array}$ & $\begin{array}{l}13.749^{a} \\
14.657 \\
120\end{array}$ & $\begin{array}{l}4 \\
4\end{array}$ & $\begin{array}{l}.008 \\
.005\end{array}$ \\
\hline
\end{tabular}

a. 3 cells ( $33.3 \%)$ have expected count less than 5 . The minimum expected count is 3.00.

While choosing colour of curtain $25 \%$ doctors opted blue, $12.5 \%$ selected vibrant while most (62.5\%) selected white as their most preferred colour. Among nurses most of them opted for blue, $7.5 \%$ chose vibrant colour while $30 \%$ chose white. Most of the patient's preferred blue colour $46.7 \%$, $45.8 \%$ chose white while $7.5 \%$ chose vibrant. In total blue was the most preferred colour (46.7\%) followed by white $(45.8 \%)$ followed by vibrant colour(7.5\%). Result was not significant.

4. Which colour of linen (bed sheets) would you prefer on the operating table in dermatology OT? White/ blue/ green/ black/ vibrant colour. 


\begin{tabular}{|c|c|c|c|c|c|}
\hline & & \multicolumn{3}{|c|}{ TYPE OF OBSERVER } & \multirow[t]{2}{*}{ Total } \\
\hline & & 1 & 2 & 3 & \\
\hline BLUE & $\begin{array}{l}\text { Count } \\
\% \text { within TYPE OF OBSERVER }\end{array}$ & $\begin{array}{l}9 \\
22.5 \%\end{array}$ & $\begin{array}{l}5 \\
12.5 \%\end{array}$ & $\begin{array}{l}5 \\
12.5 \%\end{array}$ & $\begin{array}{l}19 \\
15.8 \%\end{array}$ \\
\hline \multirow{2}{*}{ GREEN } & Count & 22 & 10 & 7 & 39 \\
\hline & $\%$ within TYPE OF OBSERVER & $55.0 \%$ & $25.0 \%$ & $17.5 \%$ & $32.5 \%$ \\
\hline \multirow{4}{*}{ WHITE } & Count & & 25 & 28 & 62 \\
\hline & $\%$ within TYPE OF OBSERVER & $22.5 \%$ & $62.5 \%$ & $70.0 \%$ & $51.7 \%$ \\
\hline & Count & & & 40 & 120 \\
\hline & $\%$ within TYPE OF OBSERVER & $100.0 \%$ & $100.0 \%$ & $100.0 \%$ & $100.0 \%$ \\
\hline
\end{tabular}

Chi-Square Tests
\begin{tabular}{|l|l|l|l|}
\hline & Value & df & $\begin{array}{l}\text { Asymp. Sig. (2- } \\
\text { sided) }\end{array}$ \\
\hline Pearson Chi-Square & $21.473^{\mathrm{a}}$ & 4 & .000 \\
Likelihood Ratio & 22.392 & 4 & .000 \\
N of Valid Cases & 120 & & \\
\hline
\end{tabular}

a. 0 cells $(0.0 \%)$ have expected count less than 5 . The minimum expected count is 6.33 .

While choosing colour of linen (bed sheets) most doctors chose green colour $55 \%$. Blue and white shared equal percentage among doctors $(22.5 \%) .62 .5 \%$ nurses chose white $(62.5 \%)$ as their most preferred colour followed by green $(25 \%)$ and blue $(12.5 \%)$. Patients preferred white $(70 \%)$ as colour of the linen followed by green $(17.5 \%)$ and blue $(12.5 \%)$. White was most common colour $(51.7 \%)$ followed by green $(32.5 \%)$.
White colour was mostly chosen by patients (70\%). Result was significant (.000).

5. Which colour of furniture including cabinets / laminates would you White/blue/green/black/vibrant colour/brown.

\begin{tabular}{|c|c|c|c|c|}
\hline \multicolumn{5}{|l|}{ Crosstab } \\
\hline & & & \multicolumn{2}{|c|}{ TYPE OF OBSERV } \\
\hline & & & 1 & 2 \\
\hline BLUE & \multicolumn{2}{|l|}{ Count } & $\begin{array}{l}0 \\
0.0 \%\end{array}$ & $\begin{array}{l}0 \\
0.0 \%\end{array}$ \\
\hline \multirow{3}{*}{ BROWN } & \multicolumn{2}{|l|}{ Count } & 40 & 40 \\
\hline & \multicolumn{2}{|c|}{$\begin{array}{l}\% \text { within TYPE OF OBSERVER } \\
\text { Count }\end{array}$} & $\begin{array}{l}100.0 \% \\
40\end{array}$ & $\begin{array}{l}100 . \\
40\end{array}$ \\
\hline & \multicolumn{2}{|c|}{$\%$ within TYPE OF OBSERVER } & $100.0 \%$ & 100. \\
\hline \multicolumn{5}{|l|}{ Chi-Square Tests } \\
\hline & Value & df & \multicolumn{2}{|c|}{\begin{tabular}{|lll}
$\begin{array}{l}\text { Asymp. } \\
\text { sided })\end{array}$ & Sig. & (2- \\
\end{tabular}} \\
\hline $\begin{array}{l}\text { Pearson Chi-Square } \\
\text { Likelihood Ratio } \\
\text { N of Valid Cases }\end{array}$ & $\begin{array}{l}4.068^{\mathrm{a}} \\
4.463 \\
120\end{array}$ & $\begin{array}{l}2 \\
2\end{array}$ & \multicolumn{2}{|l|}{$\begin{array}{l}.131 \\
.107\end{array}$} \\
\hline
\end{tabular}

a. 3 cells $(50.0 \%)$ have expected count less than 5 . The minimum expected count is .67 .

All (100\%) doctors and nurses preferred brown colour for furniture. Blue by preferred by $5 \%$ patients only. In total brown selected the most making $98.3 \%$ followed by blue (1.7\%). Result was not significant. Green, white, black or vibrant colour was chosen by none.
6. Would you prefer to have provision of windows or ventilator in dermatology OT for natural light? Yes/No

Crosstab

\begin{tabular}{|ll|l|l|l|l|}
\hline & \multicolumn{2}{|l|}{ TYPE OF OBSERVER } & Total \\
\cline { 3 - 5 } & & 1 & 2 & 3 & \\
\hline \multirow{2}{*}{ YES } & Count & 40 & 40 & 40 & 120 \\
& \% within TYPE OF OBSERVER & $100.0 \%$ & $100.0 \%$ & $100.0 \%$ & $100.0 \%$ \\
& Count & 40 & 40 & 40 & 120 \\
& \% within TYPE OF OBSERVER & $100.0 \%$ & $100.0 \%$ & $100.0 \%$ & $100.0 \%$ \\
\hline
\end{tabular}


Chi-Square Tests

\begin{tabular}{|l|l|}
\hline & Value \\
\hline $\begin{array}{l}\text { Pearson Chi-Square } \\
\text { N of Valid Cases }\end{array}$ & $\dot{a}^{\mathrm{a}}$ \\
\hline
\end{tabular}

a. No statistics are computed because 6 is a constant.

All (100\%) preferred provision of windows/ ventilator in OT for natural light. Result was significant.

7. Does display of instruments in transparent cupboard shelves or kept openly on slabs /shelves in dermatology OT generate apprehension on visualising the same? Yes/No

Crosstab
\begin{tabular}{|ll|l|l|l|l|}
\hline & & \multicolumn{5}{l|}{ TYPE OF OBSERVER } & Total \\
\cline { 3 - 5 } & & 1 & 2 & 3 & \\
\hline \multirow{2}{*}{ NO } & Count & 20 & 10 & 5 & 35 \\
& \% within TYPE OF OBSERVER & $50.0 \%$ & $25.0 \%$ & $12.5 \%$ & $29.2 \%$ \\
& Count & 20 & 30 & 35 & 85 \\
& YES within TYPE OF OBSERVER & $50.0 \%$ & $75.0 \%$ & $87.5 \%$ & $70.8 \%$ \\
& Count & 40 & 40 & 40 & 120 \\
& \% within TYPE OF OBSERVER & $100.0 \%$ & $100.0 \%$ & $100.0 \%$ & $100.0 \%$ \\
\hline
\end{tabular}

Chi-Square Tests

\begin{tabular}{|l|l|l|l|}
\hline & Value & $\mathrm{df}$ & $\begin{array}{l}\text { Asymp. Sig. (2- } \\
\text { sided) }\end{array}$ \\
\hline $\begin{array}{l}\text { Pearson Chi-Square } \\
\text { Likelihood Ratio }\end{array}$ & $14.118^{\mathrm{a}}$ & 2 & .001 \\
N of Valid Cases & 14.293 & 2 & .001 \\
\hline
\end{tabular}

a. 0 cells $(0.0 \%)$ have expected count less than 5 . The minimum expected count is

11.67 .

On displaying of instruments in dermatology OT $87.5 \%$ patient generated apprehension among patients while $12.5 \%$ did not.75\% nurses felt apprehension while $25 \%$ did not. Among doctors 50\% had apprehension while $50 \%$ did not. In total $70.8 \%$ had apprehension while $29.2 \%$ did not

Crosstab

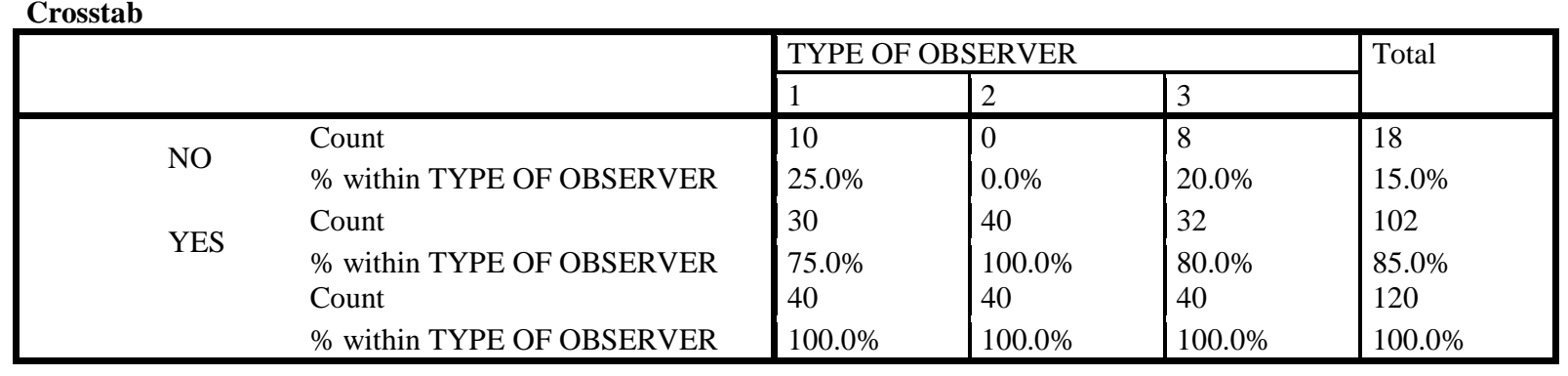

Chi-Square Tests

\begin{tabular}{|l|l|l|l|}
\hline & Value & df & $\begin{array}{l}\text { Asymp. Sig. (2- } \\
\text { sided) }\end{array}$ \\
\hline $\begin{array}{l}\text { Pearson Chi-Square } \\
\text { Likelihood Ratio } \\
\text { N of Valid Cases }\end{array}$ & $\begin{array}{l}10.980^{\mathrm{a}} \\
16.431\end{array}$ & 2 & .004 \\
120 & 2 & .000 \\
\hline
\end{tabular}

a. 0 cells $(0.0 \%)$ have expected count less than 5 . The minimum expected count is 6.00 .

On seeing greeting smile on treating dermatosurgeon's face anxiety was allayed in all nurses (100\%). Anxiety was allayed in $80 \%$ patients while $20 \%$ had no effect. Even for doctors it was important as $75 \%$ had their anxiety allayed while $25 \%$ doctors remained unaffected. In total $85.0 \%$ had apprehension on seeing the instruments. Result was significant (0.001).

8. Is your nervousness and anxiety allayed on visualising a greeting smile on the face of treating dermatosurgeon? Yes / No

were relieved on seeing greeting smile on dermatosurgeon's face. Result was significant (0.004).

9. Which fragrance / room freshener is preferred by you to be used in the OT room? Floral / Cologne / Fruity / Any Other 


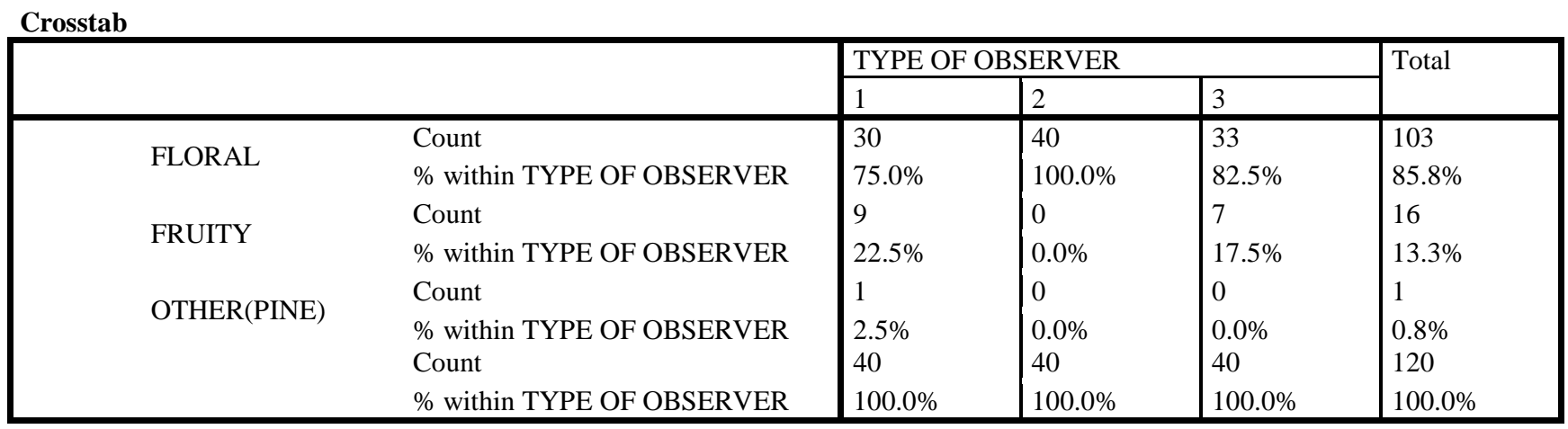

Chi-Square Tests

\begin{tabular}{|l|l|l|l|}
\hline & Value & df & $\begin{array}{l}\text { Asymp. Sig. (2- } \\
\text { sided) }\end{array}$ \\
\hline $\begin{array}{l}\text { Pearson Chi-Square } \\
\text { Likelihood Ratio }\end{array}$ & $\begin{array}{l}11.909^{\mathrm{a}} \\
16.934\end{array}$ & 4 & .018 \\
N of Valid Cases & 120 & .002 \\
\hline
\end{tabular}

a. 3 cells $(33.3 \%)$ have expected count less than 5 . The minimum expected count is .33 .

Aroma in the OT is also important. Most doctors preferred a floral $(75 \%)$ smell followed by fruity odour(22.5\%) and other smell (2.5\%). Among sisters all preferred fruity smell. $85.8 \%$ patient's preferred floral smell followed by fruity smell (13.3\%).in total $85.8 \%$ preferred floral smell

\begin{tabular}{|c|c|c|c|c|}
\hline \multicolumn{5}{|l|}{ Crosstab } \\
\hline & & & \multicolumn{2}{|c|}{ TYPE OF OBSERVER } \\
\hline & & & 1 & 2 \\
\hline NO & Count & SERYER & $\begin{array}{l}25 \\
625 \%\end{array}$ & $\begin{array}{l}2 \\
50 \%\end{array}$ \\
\hline \multirow{4}{*}{ YES } & \multirow{3}{*}{\multicolumn{2}{|c|}{$\begin{array}{l}\text { Count } \\
\% \text { within TYPE OF OBSERVER } \\
\text { Count }\end{array}$}} & & \\
\hline & & & $37.5 \%$ & $95.0 \%$ \\
\hline & & & & \\
\hline & \multicolumn{2}{|c|}{$\%$ within TYPE OF OBSERVER } & $100.0 \%$ & $100.0 \%$ \\
\hline \multicolumn{5}{|l|}{ Chi-Square Tests } \\
\hline & Value & df & \multicolumn{2}{|c|}{$\begin{array}{l}\begin{array}{l}\text { Asymp. } \\
\text { sided })\end{array} \\
\end{array}$} \\
\hline $\begin{array}{l}\text { Pearson Chi-Square } \\
\text { Likelihood Ratio } \\
\mathrm{N} \text { of Valid Cases }\end{array}$ & $\begin{array}{l}29.212^{\mathrm{a}} \\
33.942 \\
120\end{array}$ & $\begin{array}{l}2 \\
2\end{array}$ & \multicolumn{2}{|l|}{$\begin{array}{l}.000 \\
.000\end{array}$} \\
\hline
\end{tabular}

a. 0 cells $(0.0 \%)$ have expected count less than 5 . The minimum expected count is 14.33 .

Maximum doctors $62.5 \%$ were not affected by characteristic hospital smell/smell of latex glove/smell of antiseptics and medications. However $95 \%$ nurses were affected followed by patients $60 \%$. In total most $(64.2 \%)$

\begin{tabular}{|ll}
\hline Crosstab & \\
\hline & \\
\hline \multirow{2}{*}{ NO } & Count \\
& \% within TYPE OF OBSERVER \\
& Count \\
& YES within TYPE OF OBSERVER \\
& Count \\
& \% within TYPE OF OBSERVER \\
&
\end{tabular}

followed by fruity smell (13.3\%) and other smell (0.8\%). Cologne was selected by none. Result was not significant.

10. Do you prefer to avoid the characteristic hospital smell/smell of latex glove/smell of antiseptics and medications, if given a choice? Yes / No

\begin{tabular}{|l|l|l|l|}
\multicolumn{2}{|l|}{ TYPE OF OBSERVER } & Total \\
\cline { 1 - 3 } 1 & 2 & 3 & \\
\hline 25 & 2 & 16 & 43 \\
$62.5 \%$ & $5.0 \%$ & $40.0 \%$ & $35.8 \%$ \\
15 & 38 & 24 & 77 \\
$37.5 \%$ & $95.0 \%$ & $60.0 \%$ & $64.2 \%$ \\
40 & 40 & 40 & 120 \\
$100.0 \%$ & $100.0 \%$ & $100.0 \%$ & $100.0 \%$ \\
\hline
\end{tabular}

of them had problem with characteristic hospital smell in OT. Result was significant (0.000).

11. Would you like to hear soothing, pleasantries; calming words prior to procedure by doctor/supporting OT staff? Yes/No

\begin{tabular}{|l|l|l|l|}
\hline \multicolumn{2}{|l|}{ TYPE OF OBSERVER } & Total \\
\cline { 1 - 3 } & 2 & 3 & \\
\hline 20 & 0 & 0 & 20 \\
$50.0 \%$ & $0.0 \%$ & $0.0 \%$ & $16.7 \%$ \\
20 & 40 & 40 & 100 \\
$50.0 \%$ & $100.0 \%$ & $100.0 \%$ & $83.3 \%$ \\
40 & 40 & 40 & 120 \\
$100.0 \%$ & $100.0 \%$ & $100.0 \%$ & $100.0 \%$ \\
\hline
\end{tabular}


Chi-Square Tests

\begin{tabular}{|l|l|l|l|}
\hline & Value & df & $\begin{array}{l}\text { Asymp. Sig. (2- } \\
\text { sided) }\end{array}$ \\
\hline $\begin{array}{l}\text { Pearson Chi-Square } \\
\text { Likelihood Ratio } \\
\text { N of Valid Cases }\end{array}$ & $\begin{array}{l}48.000^{\mathrm{a}} \\
52.683\end{array}$ & 2 & .000 \\
\hline
\end{tabular}

a. 0 cells $(0.0 \%)$ have expected count less than 5 . The minimum expected count is 6.67 .

Hearing calming words by doctors before surgery was appreciated by all nurses and patients (100\%). Overall $83.3 \%$ wanted to hear soothing words. Result was significant (0.000).
12. Which type / nature of background music will impart a soothing effect on your mind prior and during the operative procedure?

crosstab

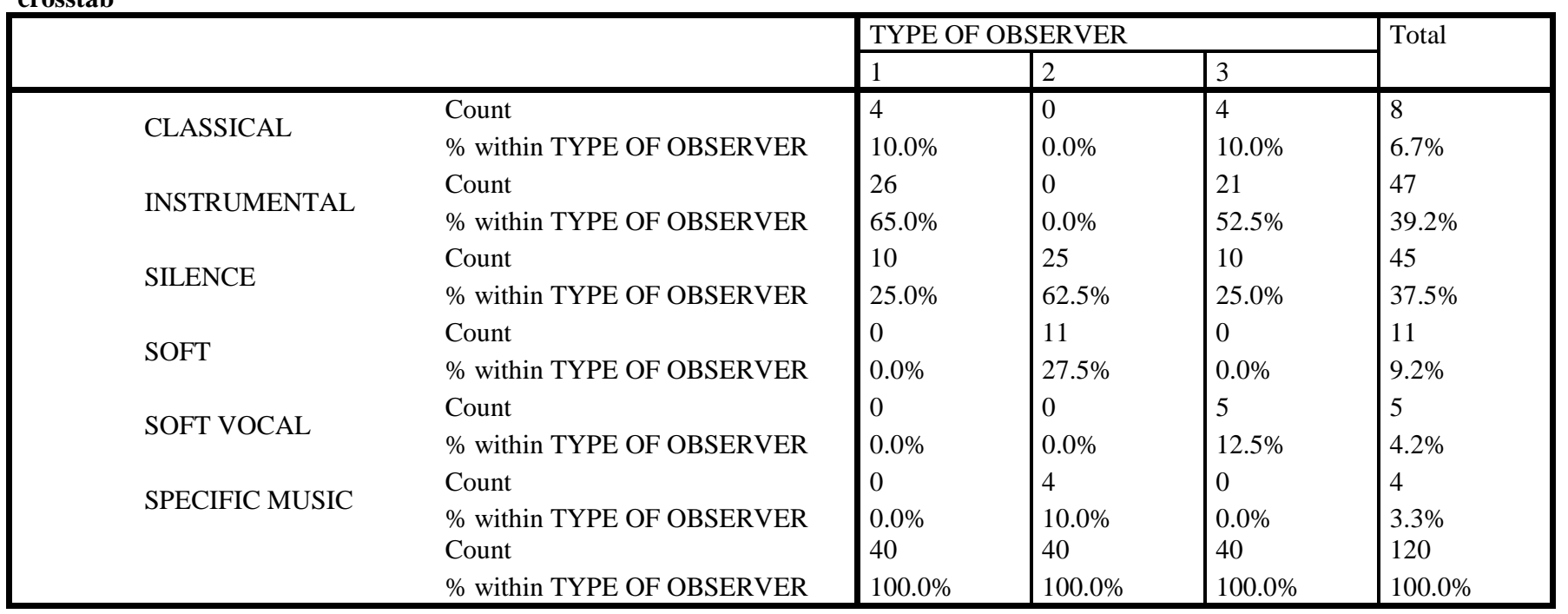

Chi-Square Tests

\begin{tabular}{|l|l|l|l|}
\hline & Value & df & $\begin{array}{l}\text { Asymp. Sig. (2- } \\
\text { sided) }\end{array}$ \\
\hline $\begin{array}{l}\text { Pearson Chi-Square } \\
\text { Likelihood Ratio } \\
\text { N of Valid Cases }\end{array}$ & $\begin{array}{l}78.298^{\mathrm{a}} \\
98.401\end{array}$ & 10 & .000 \\
120 & 10 & .000 \\
\hline
\end{tabular}

a. 12 cells $(66.7 \%)$ have expected count less than 5 . The minimum expected count is 1.33 .

When $65 \%$ doctors asked about background music wanted instrumental music followed by silence (25\%) and classical (10\%). Soft/ soft vocal / specific music was selected by none of the doctors. Most nurses preferred silence $62.5 \%$ followed by soft $(27.5 \%)$ and specific music (10\%). Most patients wanted to hear instrumental music followed by silence (10\%) and soft vocal $(12.5 \%)$. In total most of them wanted instrumental $39.2 \%$ followed by silence $(37.5 \%)$ followed by soft $(9.2 \%)$, classical $(6.7 \%)$, soft vocal $(4.2 \%)$ and specific music (3.3\%). Result was significant (0.000).

13. Does behaviour, general non-specific mutual discussion, unnecessary casual comments, lack of apparent concern or negative work attitude of supporting staff cause apprehension in your mind during the procedure? Yes/No

\begin{tabular}{|c|c|c|c|c|c|}
\hline & & \multicolumn{3}{|c|}{ TYPE OF OBSERVER } & \multirow[t]{2}{*}{ Total } \\
\hline & & 1 & 2 & 3 & \\
\hline $\begin{array}{l}\text { NO } \\
\text { YES }\end{array}$ & $\begin{array}{l}\text { Count } \\
\% \text { within TYPE OF OBSERVER } \\
\text { Count } \\
\% \text { within TYPE OF OBSERVER } \\
\text { Count } \\
\% \text { within TYPE OF OBSERVER }\end{array}$ & $\begin{array}{l}10 \\
25.0 \% \\
30 \\
75.0 \% \\
40 \\
100.0 \%\end{array}$ & $\begin{array}{l}2 \\
5.0 \% \\
38 \\
95.0 \% \\
40 \\
100.0 \%\end{array}$ & $\begin{array}{l}0 \\
0.0 \% \\
40 \\
100.0 \% \\
40 \\
100.0 \%\end{array}$ & $\begin{array}{l}12 \\
10.0 \% \\
108 \\
90.0 \% \\
120 \\
100.0 \%\end{array}$ \\
\hline
\end{tabular}


Chi-Square Tests

\begin{tabular}{|l|l|l|l|}
\hline & Value & df & $\begin{array}{l}\text { Asymp. Sig. (2- } \\
\text { sided) }\end{array}$ \\
\hline $\begin{array}{l}\text { Pearson Chi-Square } \\
\text { Likelihood Ratio } \\
\text { N of Valid Cases }\end{array}$ & $\begin{array}{l}15.556^{\mathrm{a}} \\
17.152\end{array}$ & 2 & .000 \\
120 & 2 & .000 \\
\hline
\end{tabular}

a. 3 cells $(50.0 \%)$ have expected count less than 5 . The minimum expected count is 4.00.

Most of them were disturbed( $90 \%)$ by behaviour, general non-specific mutual discussion, unnecessary casual comments, lack of apparent concern and negative work attitude of supporting staff in dermatology OT. In this all patients were affected (100\%) while 95\% nursed and $75 \%$ doctors were affected. Result was significant (0.000).
14. Before entering the OT which light refreshment(s) would you prefer prior to procedure? Water/tea/coffee/juice/soft drink/any other

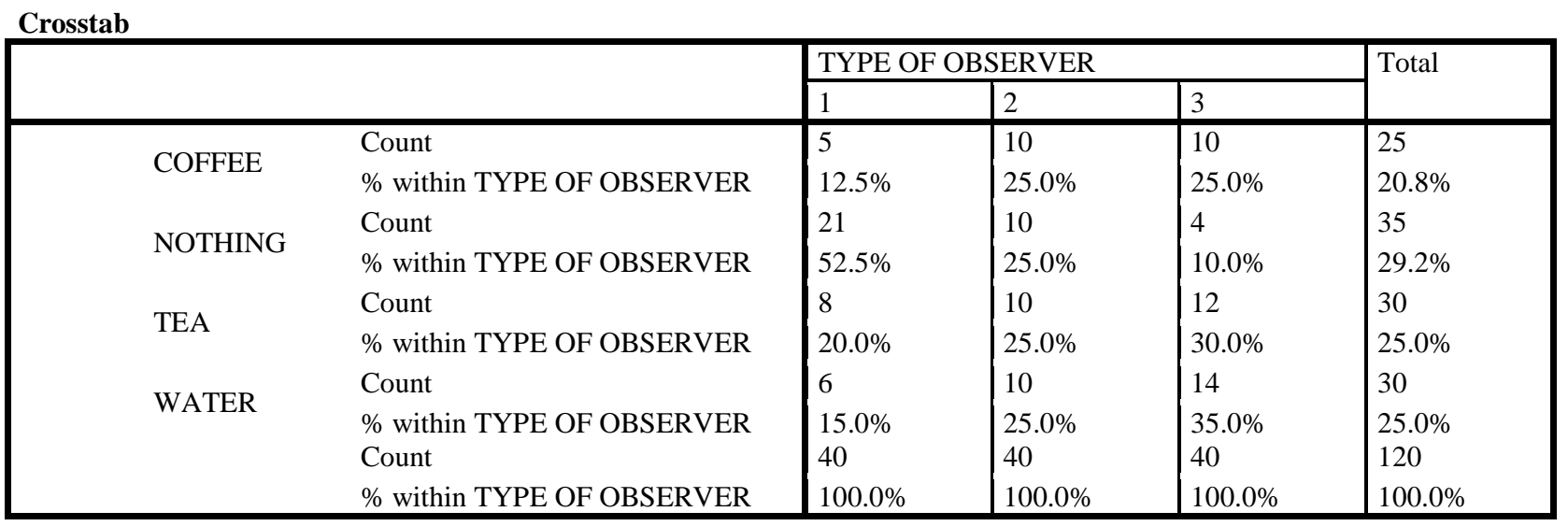

Chi-Square Tests

\begin{tabular}{|l|l|l|l|}
\hline & Value & df & $\begin{array}{l}\text { Asymp. Sig. (2- } \\
\text { sided) }\end{array}$ \\
\hline $\begin{array}{l}\text { Pearson Chi-Square } \\
\begin{array}{l}\text { Likelihood Ratio } \\
\text { N of Valid Cases }\end{array}\end{array}$ & $\begin{array}{l}18.743^{\mathrm{a}} \\
19.322\end{array}$ & 6 & .005 \\
120 & 6 & .004 \\
\hline
\end{tabular}

a. 0 cells $(0.0 \%)$ have expected count less than 5 . The minimum expected count is 8.33 .

For light refreshment(s) prior to procedure most doctors preferred nothing $52.5 \%$ followed by tea $(20 \%)$, water $(15 \%)$ and coffee $(12.5 \%)$. Nurses had equal choice for all refreshments $(25 \%)$. Patients mostly preferred water $35 \%$ followed by tea (30\%) and coffee (25\%). In total most of them Crosstab preferred nothing followed by tea $(25 \%)$ and water (25\%). Result was significant (0.000).

15. Do you prefer to eat any refreshment or snack in post-operative period - Yes / No

\begin{tabular}{|c|c|c|c|c|c|}
\hline & & \multicolumn{3}{|c|}{ TYPE OF OBSERVER } & \multirow[t]{2}{*}{ Total } \\
\hline & & 1 & 2 & 3 & \\
\hline $\begin{array}{l}\text { NO } \\
\text { YES }\end{array}$ & $\begin{array}{l}\text { Count } \\
\% \text { within TYPE OF OBSERVER } \\
\text { Count } \\
\% \text { within TYPE OF OBSERVER } \\
\text { Count } \\
\% \text { within TYPE OF OBSERVER }\end{array}$ & $\begin{array}{l}20 \\
50.0 \% \\
20 \\
50.0 \% \\
40 \\
100.0 \%\end{array}$ & $\begin{array}{l}0 \\
0.0 \% \\
40 \\
100.0 \% \\
40 \\
100.0 \%\end{array}$ & $\begin{array}{l}15 \\
37.5 \% \\
25 \\
62.5 \% \\
40 \\
100.0 \%\end{array}$ & $\begin{array}{l}35 \\
29.2 \% \\
85 \\
70.8 \% \\
120 \\
100.0 \%\end{array}$ \\
\hline
\end{tabular}

\begin{tabular}{|l|l|l|l|}
\hline Chi-Square Tests & Value & df & $\begin{array}{l}\text { Asymp. Sig. (2- } \\
\text { sided) }\end{array}$ \\
\hline Pearson Chi-Square & $26.218^{\mathrm{a}}$ & 2 & .000 \\
Likelihood Ratio & 36.496 & 2 & .000 \\
N of Valid Cases & 120 & & \\
\hline
\end{tabular}

a. 0 cells $(0.0 \%)$ have expected count less than 5 . The minimum expected count is 11.67. 
For eating refreshments or snack in post-operative period all nurses said yes $(100 \%) .62 .5 \%$ patients wanted refreshment. While among doctors the opinion was equally divided $(50 \%)$. In total most of them wanted refreshments $70.8 \%$. Result was significant (0.000).
16. Do you prefer a dehumified, cool and pleasant environment rather than hot and humid environment, which is usually found in tropical country like India? Yes/No

Crosstab
\begin{tabular}{|l|l|}
\hline \multicolumn{1}{|c|}{ YES } & $\begin{array}{l}\text { Count } \\
\% \text { within TYPE OF OBS } \\
\text { Count } \\
\% \text { within TYPE OF OBS }\end{array}$ \\
\hline Chi-Square Tests & Value \\
\hline &.$^{\text {a }}$ \\
\hline $\begin{array}{l}\text { Pearson Chi-Square } \\
\text { N of Valid Cases }\end{array}$ \\
\hline
\end{tabular}

a. No statistics are computed because 16 is a constant.

Everyone (100\%) preferred dehumified, cool and pleasant environment rather than hot and humid environment in dermatology OT.

17. Do you prefer additional presence of fan in air conditioned room for fresh blow of air to avoid feeling of suffocation? Yes/No

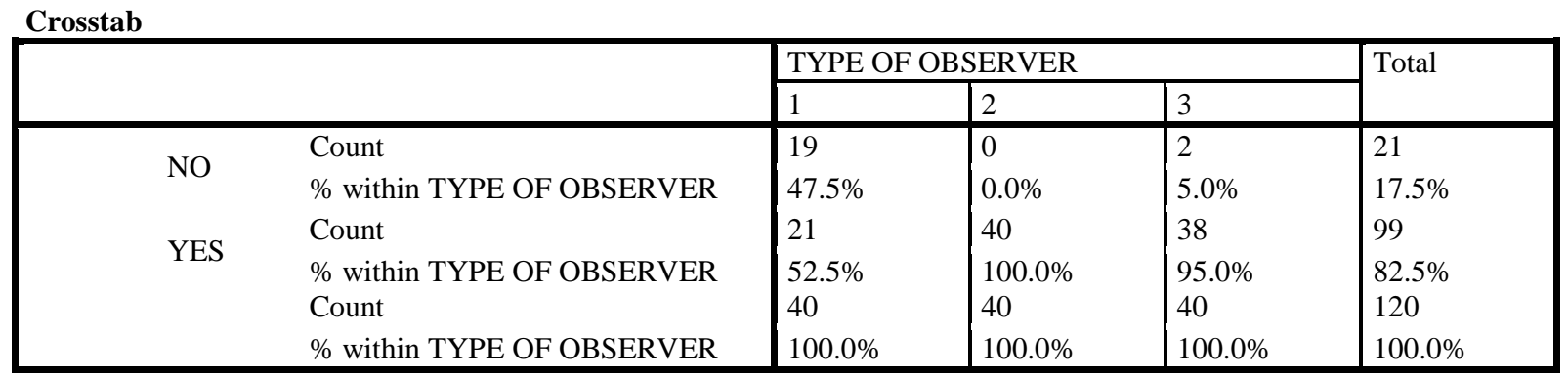

Chi-Square Tests
\begin{tabular}{|l|l|l|l|}
\hline & Value & df & $\begin{array}{l}\text { Asymp. Sig. (2- } \\
\text { sided) }\end{array}$ \\
\hline $\begin{array}{l}\text { Pearson Chi-Square } \\
\text { Likelihood Ratio }\end{array}$ & $\begin{array}{l}37.749^{\mathrm{a}} \\
40.061\end{array}$ & 2 & .000 \\
N of Valid Cases & 120 & .000 & \\
\hline
\end{tabular}

a. 0 cells $(0.0 \%)$ have expected count less than 5 . The minimum expected count is 7.00.

Most $(82.5 \%)$ of them wanted additional presence of fan in air conditioned room for fresh blow of air to avoid feeling of suffocation in dermatology OT. In this all nurses (100\%), 95\% patients and $52.5 \%$ doctors preferred additional fan. Result was significant (0.000).
18. Do you prefer to avoid excessive use of topical chemicals and medications during the procedure, apart from those absolutely necessary for the said procedure on your skin - Yes / No

Crosstab

\begin{tabular}{|ll|l|l|l|l|}
\hline & & \multicolumn{2}{l|}{ TYPE OF OBSERVER } & Total \\
\cline { 3 - 5 } & & 1 & 2 & 3 & \\
\hline \multirow{2}{*}{ NO } & Count & 13 & 0 & 2 & 15 \\
& \% within TYPE OF OBSERVER & $32.5 \%$ & $0.0 \%$ & $5.0 \%$ & $12.5 \%$ \\
& Count & 27 & 40 & 38 & 105 \\
& \%ES within TYPE OF OBSERVER & $67.5 \%$ & $100.0 \%$ & $95.0 \%$ & $87.5 \%$ \\
& Count & 40 & 40 & 40 & 120 \\
& \% within TYPE OF OBSERVER & $100.0 \%$ & $100.0 \%$ & $100.0 \%$ & $100.0 \%$ \\
\hline
\end{tabular}


Chi-Square Tests

\begin{tabular}{|l|l|l|l|}
\hline & Value & df & $\begin{array}{l}\text { Asymp. Sig. (2- } \\
\text { sided) }\end{array}$ \\
\hline $\begin{array}{l}\text { Pearson Chi-Square } \\
\text { Likelihood Ratio } \\
\text { N of Valid Cases }\end{array}$ & $\begin{array}{l}22.400^{\mathrm{a}} \\
24.097\end{array}$ & 2 & .000 \\
120 & 2 & .000 \\
\hline
\end{tabular}

a. 0 cells $(0.0 \%)$ have expected count less than 5 . The minimum expected count is 5.00 .

Most of them (87.5\%) wanted to avoid excessive use of topical chemicals and medications during the procedure. In this all nurses (100\%),95\% patients and $67.5 \%$ doctors wanted to avoid . Result was significant $(0.000)$.
19. Do you feel discomfort when instruments, surgical instruments are kept either on the abdomen or other body parts over the drapes during the surgical procedure - Yes / No

\begin{tabular}{|c|c|c|c|c|c|}
\hline & & \multicolumn{3}{|c|}{ TYPE OF OBSERVER } & \multirow[t]{2}{*}{ Total } \\
\hline & & 1 & 2 & 3 & \\
\hline $\begin{array}{l}\text { NO } \\
\text { YES }\end{array}$ & $\begin{array}{l}\text { Count } \\
\% \text { within TYPE OF OBSERVER } \\
\text { Count } \\
\% \text { within TYPE OF OBSERVER } \\
\text { Count } \\
\% \text { within TYPE OF OBSERVER }\end{array}$ & $\begin{array}{l}19 \\
47.5 \% \\
21 \\
52.5 \% \\
40 \\
100.0 \% \\
\end{array}$ & $\begin{array}{l}0 \\
0.0 \% \\
40 \\
100.0 \% \\
40 \\
100.0 \% \\
\end{array}$ & $\begin{array}{l}0 \\
0.0 \% \\
40 \\
100.0 \% \\
40 \\
100.0 \% \\
\end{array}$ & $\begin{array}{l}19 \\
15.8 \% \\
101 \\
84.2 \% \\
120 \\
100.0 \%\end{array}$ \\
\hline
\end{tabular}

Chi-Square Tests

\begin{tabular}{|l|l|l|l|}
\hline & Value & $\mathrm{df}$ & $\begin{array}{l}\text { Asymp. Sig. (2- } \\
\text { sided) }\end{array}$ \\
\hline $\begin{array}{l}\text { Pearson Chi-Square } \\
\text { Likelihood Ratio } \\
\text { N of Valid Cases }\end{array}$ & $\begin{array}{l}45.149^{\mathrm{a}} \\
49.503 \\
120\end{array}$ & 2 & .000 \\
\hline
\end{tabular}

a. 0 cells $(0.0 \%)$ have expected count less than 5 . The minimum expected count is 6.33 .

All nurses and patients (100\%) felt uncomfortable when instruments, surgical instruments were kept on the abdomen or other body parts during the surgical procedure. While only $52.5 \%$ doctors felt discomfort for the same. In total $84.2 \%$ people were not comfortable. Result was significant (0.000). Patients have feeling of suffocation when surgical instruments are kept either on the abdomen or other body parts. They also have feeling of slipping instrument making them uncomfortable. Many times instruments fall down, instrument count is missed by sister and surgery is hampered.

20. During procedure which position do you find more comfortable? Sitting on chair/ Lying on table

\begin{tabular}{|c|c|c|c|c|c|}
\hline & & \multicolumn{3}{|c|}{ TYPE OF OBSERVER } & \multirow[t]{2}{*}{ Total } \\
\hline & & 1 & 2 & 3 & \\
\hline $\begin{array}{l}\text { LYING } \\
\text { SITTING }\end{array}$ & $\begin{array}{l}\text { Count } \\
\text { \% within TYPE OF OBSERVER } \\
\text { Count } \\
\text { \% within TYPE OF OBSERVER } \\
\text { Count } \\
\text { \% within TYPE OF OBSERVER }\end{array}$ & $\begin{array}{l}29 \\
72.5 \% \\
11 \\
27.5 \% \\
40 \\
100.0 \%\end{array}$ & $\begin{array}{l}32 \\
80.0 \% \\
8 \\
20.0 \% \\
40 \\
100.0 \%\end{array}$ & $\begin{array}{l}27 \\
67.5 \% \\
13 \\
32.5 \% \\
40 \\
100.0 \%\end{array}$ & $\begin{array}{l}88 \\
73.3 \% \\
32 \\
26.7 \% \\
120 \\
100.0 \%\end{array}$ \\
\hline
\end{tabular}

Chi-Square Tests

\begin{tabular}{|c|c|c|c|}
\hline & Value & $\overline{d f}$ & $\begin{array}{lll}\begin{array}{l}\text { Asymp. } \\
\text { sided) }\end{array} & \text { Sig. } & \text { (2- } \\
\end{array}$ \\
\hline $\begin{array}{l}\text { Pearson Chi-Square } \\
\text { Likelihood Ratio } \\
\mathrm{N} \text { of Valid Cases }\end{array}$ & $\begin{array}{l}1.619^{\mathrm{a}} \\
1.647 \\
120\end{array}$ & $\begin{array}{l}2 \\
2\end{array}$ & $\begin{array}{l}.445 \\
.439\end{array}$ \\
\hline
\end{tabular}

a. 0 cells $(0.0 \%)$ have expected count less than 5 . The minimum expected count is

10.67 . 
Lying was found to be more comfortable during procedure by most $73.3 \%$. In this $80 \%$ nurses, $72.5 \%$ doctors and $67.5 \%$ patients preferred lying. Result was not significant.

\section{While undergoing procedure which position do you find more comfortable? Supine/prone/lateral}

All (100\%) preferred supine position during procedure.

\section{Discussion}

Colour is a powerful communication tool and can be used to signal action, influence mood, and even influence physiological reactions. Feelings about colour are often deeply personal and rooted in your own experience or culture. For example, while the colour white is used in many Western countries to represent purity and innocence, it is seen as a symbol of mourning in many Eastern countries. While perceptions of colour are somewhat subjective, there are some colour effects that have universal meaning. Colour psychology suggests that various shades can have a wide range of effects, from boosting our moods to causing anxiety (Table 2 ). For example the colour white can feel fresh and clean. The colour is often used to evoke a sense of youth and modernity. People often describe blue as the colour of stability and safety. Driving a blue car might indicate that you are dependable and trustworthy. A person might prefer brighter, more attention-getting colours when they are younger, but find themselves drawn to more traditional colours as they grow older. Room colours can also be used to evoke specific moods, such as painting a bedroom a soft green to create a peaceful mood [5]. Colour psychology is mainly distinguished by two categories of colours: warm and cool. Warmer colours stimulate feelings of anger, hostility as well as comfort and warmth. Cooler colours, on the other hand, help instil feelings of sadness but calmness as well. All cool colours like green and blue are easy on the eye muscles and hence facilitate their relaxation. Offices having warmer coloured hues such as browns and golds actually make people feel warmer while those with lighter colours like pale blue or white make people feel cooler. A scientific experiment helps demonstrate colour psychology better: Two study groups were each given sleeping pills. The only difference was that one group was given a "blue pill" while another was given a "pink pill". $72 \%$ of those who took the blue pill reported feeling sleepy as compared to only $32 \%$ of those who took the pink one. This could be because of the fact that blue inspires calmness and serenity and is associated with water and peace [6]. Certain colours have even been associated with increased blood pressure, increased metabolism, and eyes train [5].

A great deal of thought and consideration must be given to placement and usage of colour psychology in work and home environments as it directly impacts one's physical and mental welfare [6]. Experts have found that while colour can have an influence on how we feel and act, these effects are subject to personal, cultural, and situational factors. More scientific research is needed to gain a better understanding of colour psychology [5].

Table 2: Colour psychology [5]

\begin{tabular}{|l|l|}
\hline COLOUR & COLOUR PSYCHOLOGY \\
\hline Black & Sophistication, power, mystery, formality, evil, death \\
\hline Yellow & Joy, cheerfulness, energy, warmth. caution, cowardice \\
\hline White & Freshness, hope, goodness, light, purity, cleanliness, simplicity, coolness \\
\hline Blue & Peace, stability, calmness, confidence, sincerity, affection \\
\hline Green & Life, growth, environment, healing, money, safety, relaxation, freshness \\
\hline Pink & Romance, compassion, faithfulness, beauty, love friendship, sensitivity \\
\hline Purple & Royalty, luxury, dignity, wisdom, spirituality, passion, vision, magic \\
\hline
\end{tabular}

Windows are of key importance; as well as natural light, they provide an outlook, contact with the outside and access to sunlight about which patients are extremely positive. Windows should therefore be sized and positioned to provide a view out, regardless of location, as well as a reasonable average daylight factor. Lighting has a considerable effect on the appearance of a space [1].

Healthcare professionals' empathic behaviour is an important component of quality health care. Patients' reports suggest that empathy is often lacking. Specific factors that may facilitate or inhibit empathetic behaviour have not been extensively examined [2]. Patients indicate a need to be recognized and treated as individuals by hospital staff. They are concerned with their illnesses and conditions and want to be kept informed.An atmosphere respectful of the individual patient should be focused. Fear and anxiety associated with illness can be as debilitating as the physical effects. Proper coordination of care can ease those feelings. Modifying external behaviour and how doctors communicate is very important. More value should be placed on talking with patients. A doctor's behaviour also serves as a model to the students, interns, and medical staff around him. The benefits of setting the behavioural tone cannot be underestimated [7].

Our mental state is influenced and often dominated by olfactory experiences that guide our senses. Whether it is an apple that looks rotten or a rose that seems freshly watered, behind the scenes it's our sense of smell that's responsible for much of those sensations [8].
An odour has no personal significance until it becomes connected to something that has meaning. The capacities for both smell and emotion are rooted in the same network of brain structures, the limbic system. The olfactory centre also interacts directly with the hippocampus, a brain area involved in the formation of new memories. Scents can have positive effects on mood, stress reduction, sleep enhancement, self-confidence, and physical and cognitive performance.

Aromas have different effects on everyone, but the natural fragrances are odours for the masses. Peppermint is generally invigorating. It increases activity in the brain area that wakes us up in the morning. Jasmine is a sleep aid. The scent increases the brain waves associated with deep sleep. Lavender is generally relaxing. Smells can affect both our short and long term moods. Creating an environment with pleasant smells which evoke positive, calm or otherwise good emotions is rather simple and can have a profound impact on our mood [8].

Any patient going into surgery will naturally feel anxious. That's why standard procedure is to give them a dose of anxiety medicine, typically hydroxyzine, which helps them relax before receiving anaesthesia [10]. However, "conversational hypnosis", does a better job than pills for relaxing patients, suggests research being presented at the ANESTHESIOLOGYTM 2015 annual meeting. According to Dr. Boselli, the study suggests that conversational hypnosis might increase patient self-reported comfort during regional anesthesia without medication, and that this effect might be objectively monitored using the ANI Analgesia/Nociception Index (ANI) [11]. 
Listening to music has been shown to improve memory functioning, increased rate of healing, improve your workouts and more. Just like listening to slow music to calm the body, music can also have a relaxing effect on the mind [12] Fast, upbeat music provide a burst of energy while exercising, slower music has been shown to be more relaxing. Classical music, in particular, can help slow the pulse and heart rate, as well as decrease levels of stress hormones [13]. Fast music increases the cortisol levels, while the slow, quiet, calm and classical music does the opposite. The blood cortisol levels of those with imminent surgery get reduced by $50 \%$ with the calming music, jointly chosen by the patient and music therapist.An emotional response of the secretion of immune-boosting hormones is induced by certain music. Music decreases the stress-related hormone cortisol, the increased levels of which weaken the immune system. Hence the chances of illness get reduced [14]. A study suggests playing soothing music to patients before anaesthetising them for surgery could calm their nerves as much as conventional anxiety drugs but with fewer side effects [15]. Listening to meditative music as a non-invasive and cost-effective strategy can help maximize efforts to promote comfort and relaxation for patients awaiting stressful procedures. Meditative music can be effective in alleviating state anxiety of patients [16]

Optimal positioning not only ensures the best possible access to the surgical site, but also prevents long-term consequences such as nerve damage or pressure ulcers. These secondary complications can delay rehabilitation and recovery. Patients have better outcomes when hospitals can avoid these secondary consequences. Surgical teams should always choose a position that maximizes surgical site access while minimizing the risk of complications. In prone positions obese patients feel suffocated. The respiratory distress compounds to surgical side effects [17].

\section{References}

1. Dalke H,Littlefair PJ,Loe DL,Camgoz N. (2004) Lighting and colour for hospital design.p.8-9.

2. Elayyan M, Rankin J, Chaarani MW. (2018) Factors affecting empathetic patient care behaviour among medical doctors and nurses: an integrative literature review. East Mediterr Health J. 24(3):311-318.

3. Scent and Emotion - How Do Smells Affect The Brain?

4. Fukui H,Toyoshima K. (2013) Influence of music on steroid hormones and the relationship between receptor polymorphisms and musical ability: a pilot study. Front Psychol.

5. Cherry K. (2019) Color Psychology: Does It Affect How You Feel?

6. What are the effects of colors on the brain?

7. Ofri D. (2017) What Patients Say, What Doctors Hear?

8. Scent and Emotion - How Do Smells Affect The Brain?

9. The Hidden Force of Fragrance.

10. Bushak L. (2015) Conversational Hypnosis': Calming Words Do More Than Pills To Reduce Anxiety In Surgery Patients.

11. Soothing words do more than pills to calm anxious patients, study shows.

12. The Benefits of Music: How the Science of Music Can Help You.

13. The Science behind the Relaxing Effects of Music.

14. The Effect of Music on Human Health and Brain Growth.

15. King AM. (2004) Soothing music calms pre-surgery patients without the side effects of drugs.

16. LeeW-L, Sung H-C, Liu S-H, Chang S-M. (2017) Meditative music listening to reduce state anxiety in patients during the uptake phase before positron emission tomography (PET) scans. Br J Radiol.

17. Optimizing Patient Positioning. 